\title{
LA EDAD DE BROCE DE SAN FERNANDO: UN MODELO DE FORMACIÓN ECONÓMICO-SOCIAL PERIFÉRICO EN LA BANDA ATLÁNTICA DE CÁDIZ
}

\author{
BRONZE AGE AT SAN FERNANDO. A PERIPHERAL SOCIO-ECONOMIC \\ FORMATION IN THE ATLANTIC COAST OF CADIZ
}

\author{
por \\ JOSÉ RAMOS MUÑOZ* , ANTONIO SÁEZ ESPLIGARES**, \\ VICENTE CASTAÑEDA FERNÁNDEZ***, JORGE CEPILLO GALVÍN ***, \\ MANUELA PÉREZ RODRÍGUEZ*** y JOSÉ MARÍA GUTIÉRREZ LÓPEZ ***. \\ ÁREA DE PREHISTORIA. FACULTAD DE FILOSOFÍA Y LETRAS. \\ UNIVERSIDAD DE CÁDIZ.
}

RESUMEN: Exponemos un balance sobre la Edad del Bronce en San Fernando. Analizamos las bases arqueológicas para el estudio de las sociedades de mediados del $\mathrm{II}^{\circ}$ milenio a.C. . Se trata de comunidades periféricas, que habitan un medio insular, donde aprovechan recursos naturales, fundamentalmente malacológicos, pero que generan una importante agricultura, existiendo una clara relación de dependencia respecto a un centro nuclear ubicado en las campiñas interiores.

\begin{abstract}
We show a summary about Bronze Age in the island of San Fernando. Archaeological basis are analyzed to study the societies of the midle of the second millennium B.C. They are peripheric communities who live in the island, where they use natural resources, fundamentally molluscs, which generate an important agriculture, so there is a clear relation of dependence with regard to a nuclear center in the interior contrysides.
\end{abstract}

\section{INTRODUCCIÓN}

Vamos a exponer el estado actual del conocimiento de la Edad del Bronce en el entorno geográfico, insular de San Fernando, analizando las bases geográficas y geomorfológicas de su ubicación, considerando los elementos arqueológicos obtenidos a partir de la prospección sistemática y de una excavación

\footnotetext{
* Profesor de Prehistoria. Universidad de Cádiz.

** Museo Histórico de San Fernando.

*** Licenciados en Historia. Universidad de Cádiz.
} 


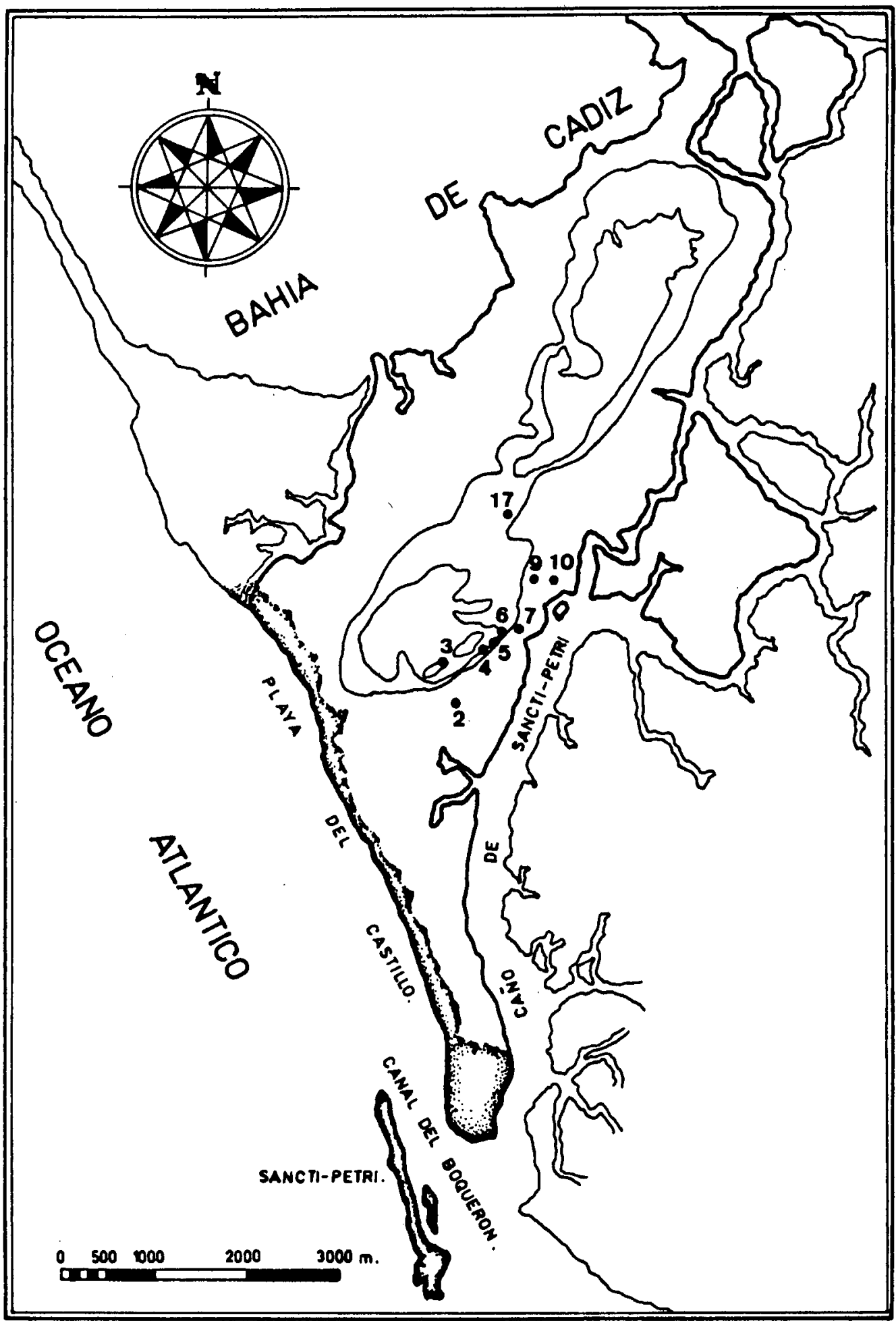

FIGURA 1. Localización de los yacimientos de la Edad del Bronce de San Fernando. 2 Eı Estanquillo. 3 Camposoto. 4 La Marquina A. 5 La Marquina B. 6 La Marquina C. 7 Pago de la Zorrera. 9 Huerta de Suraña A. 10 Huerta de Suraña B. 17 Edificio Berenguer. 
de urgencia realizada en el asentamiento de El Estanquillo. Valoramos la ubicación geográfica y un enmarque espacial de los asentamientos. Exponemos un desarrollo sucinto de las bases de la cultura material de estas poblaciones, lítica y cerámica, en un plano morfológico, y en aspiración a su interpretación funcional y contextual, para con ello exponer un ensayo de hipótesis de trabajo, sobre las relaciones de dependencia económica, social y por ende políticas, de estas comunidades insulares y costeras de la Bahía de Cádiz, respecto a centros nucleares situados en el interior, en las ricas campiñas de Medina Sidonia y Chiclana de la Frontera.

Se plantea un modelo jerárquico de poblados que acumulan excedentes agrícolas, y que controlan la producción de enclaves costeros y periféricos como los situados en San Fernando. Trabajamos aquí pues con un modelo de la «periferia» de un centro núclear en fase de definición arqueológica, pero del que comenzamos a tener hipótesis formuladas de su estructuración, para poder afirmar que el control económico y político se ejerce desde el interior hacia la costa.

Creemos, como estrategia de investigación, que desde el conocimiento de las comunidades periféricas se puede ayudar a interpretar y comprender plenamente la Historia de las formaciones económicas y sociales y valorar así ajustadamente las relaciones de los centros nucleares con sus áreas de controldominación.

Desde un plano metodológico queremos indicar nuestra concepción de la Prehistoria, como compromiso metodológico con un corpus teórico conceptual, que se configura por bases filosóficas, de la teoría de la Ciencia, concepto de Historia y posición metodológica ante la actividad de producción intelectual prehistórica. Creemos necesaria la articulación dialéctica, armónica y concreta entre el pensamiento y la producción arqueológica, configurada así como fenómeno imbricado de teoría-práctica. Nuestra posición de partida, aspira a trabajar desde modelos conceptuales-metodológicos del Materialismo Histórico.

\section{APROXIMACIÓN AL ESTUDIO DE LA EDAD DEL BRONCE DE SAN FERNANDO}

Este trabajo que aquí presentamos se enmarca en un proyecto de investigación titulado «La ocupación prehistórica de la campiña litoral y banda atlántica de Cádiz», autorizado y subvencionado por la Dirección General de Bienes Culturales de la Junta de Andalucía (Ramos et Al. , 1993).

El estudio de la Edad del Bronce cuenta con una serie de analíticas científicas, que partiendo desde el marco teórico propuesto aspira a la comprensión global de dicha formación social en su proceso histórico, previa delimitación de su marco territorial.

El estudio geográfico, geomorfológico y la reconstrucción paleoclimática está a cargo del profesor Dr. Francisco Borja (Departamento de Geografía Física-A.G.R. Universidad de Huelva) (Borja, 1992, Borja, et Al. , En prensa; Borja y Ramos,, En prensa a, b).

La excavación del asentamiento de El Estanquillo-Fase II-Estrato III (Ramos, 1990, 1991, 1993), ha posibilitado unos estudios analíticos científicos fundamentales para la comprensión de la estructura económica de esta formación económico-social. Así, la malacofauna está siendo analizada por el Dr. Alexander Menez (Gibraltar Museum). La fauna la estudia Da . Eloisa Bernáldez (Estación Biológica de Doñana. C.S.I.C. Sevilla). El estudio antropológico del enterramiento corre a cargo del Dr. José Alcazar Godoy (Laboratorio de Antropología Física. Museo Arqueológico de Sevilla). El análisis de la composición y pastas cerámicas lo realizan los profesores Dr. J. Martín Calleja y Dr. M.J. Felíu (Departamento de Química-Física. Facultad de Ciencias. Universidad de Cádiz). Una muestra metálica es estudiada por el Dr. Salvador Rovira (Museo de América). Los restos orgánicos vegetales están a cargo del profesor Dr. Javier Herrera (Departamento de Botánica. Facultad de Biología. Universidad de Sevilla). Las dataciones de Radiocarbono se analizan en la University Branch de Florida (U.S.A.). 


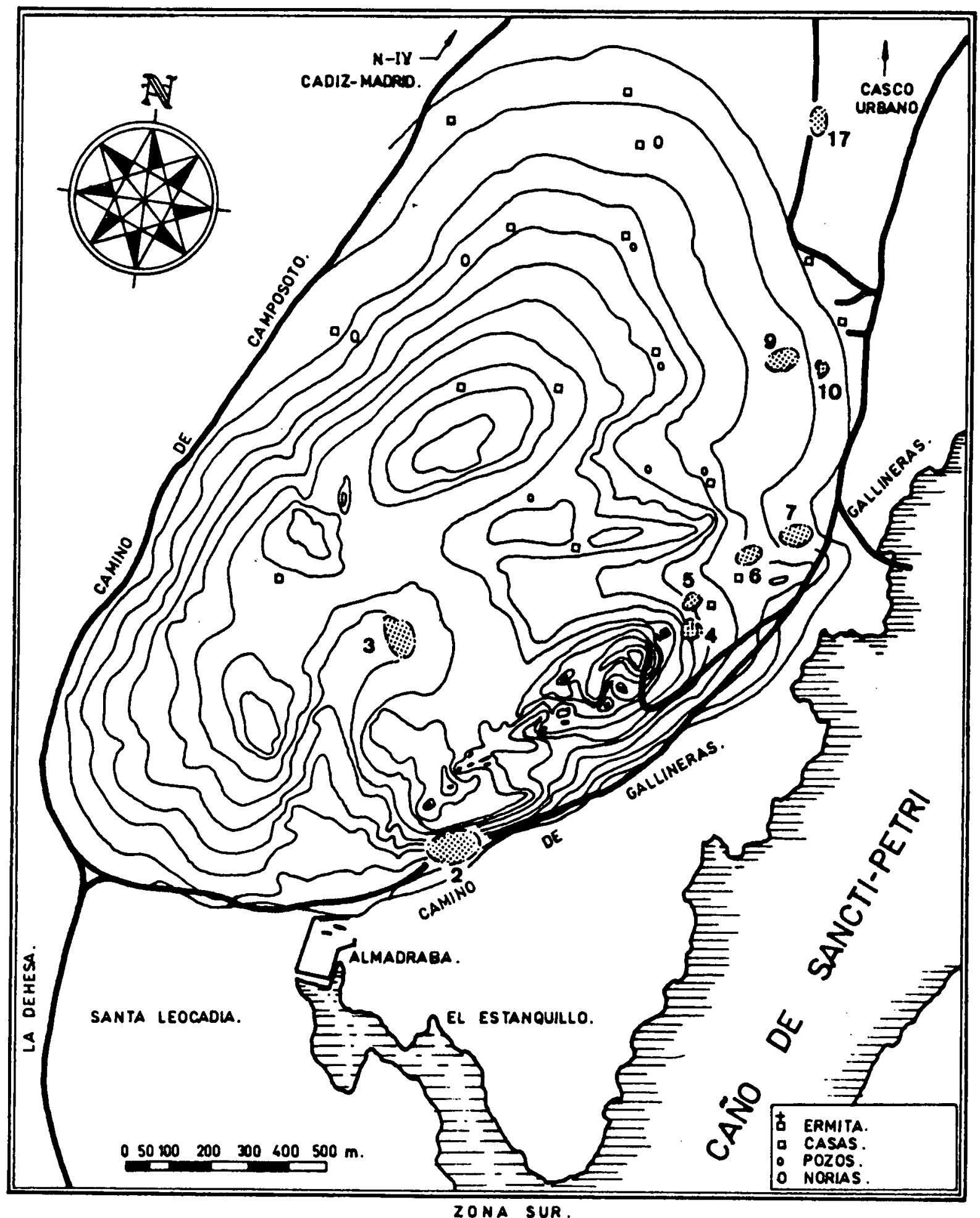

FIGURA 2. Areas de dispersión de los yacimientos de la Edad del Bronce de San Fernando. 2 El Estanquillo. 3 Camposoto. 4 La Marquina A. 5 La Marquina B. 6 La Marquina C. 7 Pago de la Zorrera. 9 Huerta de Suraña A. 10 Huerta de Suraña B. 17 Edificio Berenguer. 
Las manifestaciones de industrias líticas talladas sobre cantos, son analizadas por el profesor Dr. Enrique Vallespí (Departamento de Prehistoria y Arqueología. Universidad de Sevilla) y por el profesor Dr.José Ramos.

El estudio arqueológico corre a cargo de un equipo formado por José Ramos, Antonio Sáez, Vicente Castañeda, Jorge Cepillo, Manuela Pérez y José María Gutierrez.

Por tanto, los resultados de este trabajo corresponden a una elaboración preliminar, que debe completarse a medio plazo con la información generada por los mencionados análisis, que a su vez se contrastarán en el desarrollo del proyecto de investigación con los datos obtenidos en las campiñas del interior.

\section{APROXIMACIÓN PALEOGEOGRÁFICA}

La zona SW de Cádiz comprende paisajes característicos de alineaciones de areniscas y calcáreas agrestes, con piedemontes desarrollados y campiñas acolinadas con cerros individualizados, litorales alternantes y marismas en desembocaduras. Geológicamente se caracteriza por un predominio de unidades pertenecientes al cierre Neógeno de la cuenca inferior del Guadalquivir y al Pleistoceno.

El paisaje de San Fernando se define por la unidad del Cerro de los Mártires, de altitud máxima de 34 mts., con proyección al NW, donde se ubica en una mesa significativa la ciudad de San Fernando, a 20 mts. de altitud. Es así un paisaje abierto, de suaves laderas. El resto son marismas, dedicadas tradicionalmente a la explotación salinera y «caños» de marismas pantanosas. La banda costera cuenta con una estrecha y alargada superficie de dunas costeras. Geológicamente se documentan arenas y arcillas del Plioceno Superior-Pleistoceno, en la falda S.E. del Cerro de los Mártires, conglomerados de cantos de cuarzo y cuarcita del Pleistoceno en el área de La Dehesa y sobre todo arenas, limos y arcillas holocénicas en las marismas (Zazo, et Al. , 1987).

Petrográficamente se localizan cantos de cuarzo, cuarcita y sílex aportados en el cuaternario a modo de glacis de cobertera por los ríos Guadalete, Arillo y San Pedro (Zazo, et Al. , 1987). Estos materiales conforman la materia prima fundamental para la elaboración de artefactos prehistóricos.

La Banda Atlántica de Cádiz, en los entornos de San Fernando y Bahía de Cádiz ha sufrido importantes cambios y alteraciones morfológicas en el Holoceno. A partir de la Transgresión Flandriense el espacio del actual San Fernando se configura como isla (Gavala, 1924; Zazo, 1989; Borja, F. , 1992). Después del máximo de la subida del nivel del mar, la evolución del Holoceno conlleva importantes cambios en las condiciones paleogeográficas, durante el Tardiglacial. Se observa una etapa húmeda, que implica la génesis de Tierras Negras (Borja et $\mathrm{Al}$., en prensa), controladas desde Chipiona a Barbate en el área de nuestro estudio, datadas en Holoceno Medio (6000-3000 a.C.), quedando relacionadas con otros suelos similareas asociados al Pluvial Neolítico norafricano. Decapitando y sellando estas Tierras Negras hay evidencias de aportes coluvio-aluviales de arroyada, y/o dunas que indican un aumento de la aridez, tras el ciclo húmedo neolítico, que se acentúa en el episodio subboreal (Borja, 1992).

En relación con la ocupación prehistórica de la Bahía de Cádiz, a partir de la Edad del Cobre estamos viendo que la progresiva antropización del medio acentúa este proceso de arroyadas y de aridez, con la instauración de una agricultura intensiva de cereal, con la importancia de la ganadería mixta de bovinos y ovinos, unido todo ello a un manifiesto proceso de desforestación (Borja y Ramos, en prensa a, b).

Por tanto a mediados del II milenio a.C., el área del actual San Fernando aún se conforma como isla, en un proceso de creación de extensas llanuras mareales (Zazo, 1989), con enormes posibilidades de explotaciones económicas, agrícolas, ganaderas y de captación de recursos marinos. 


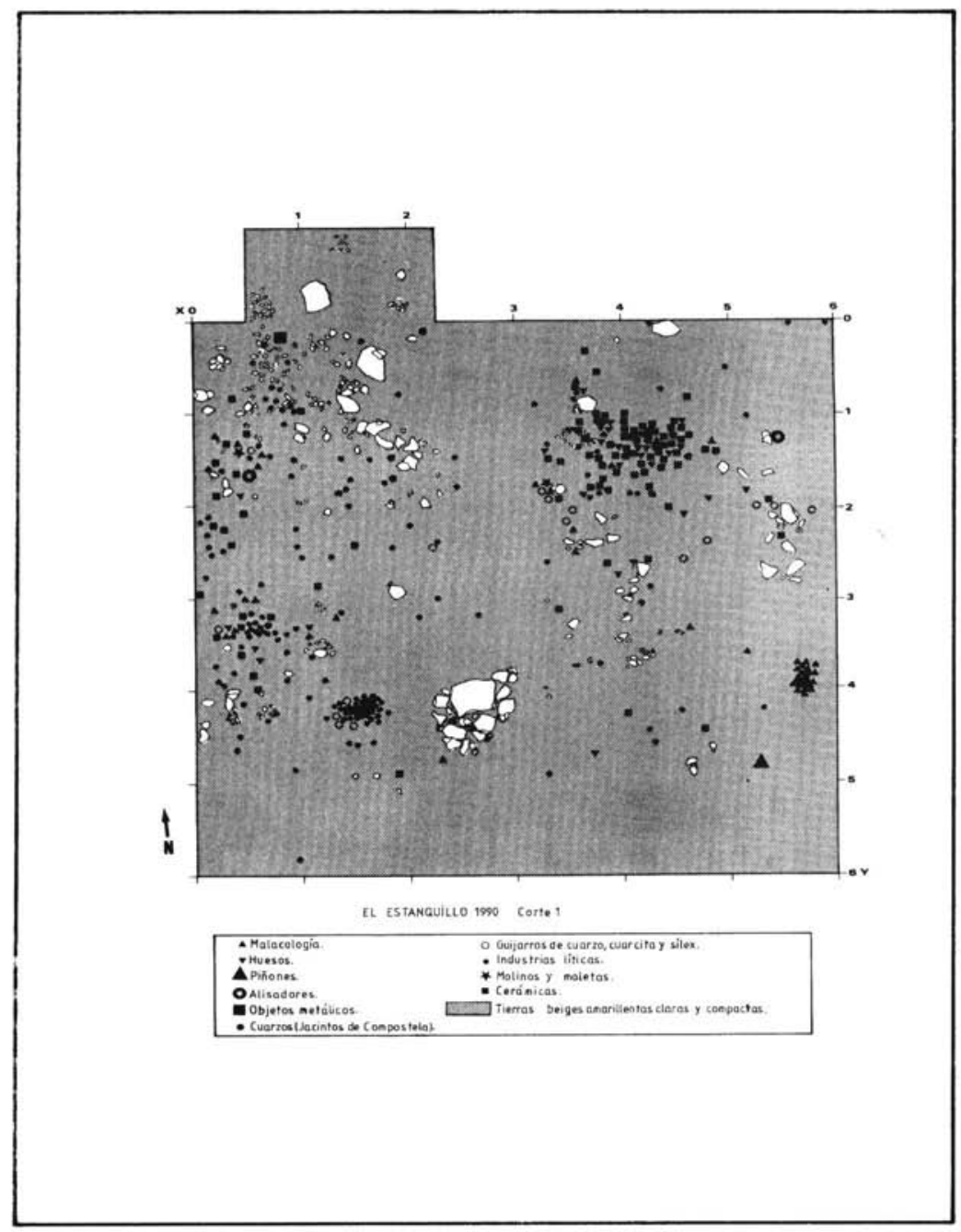

FIGURA 3. Planta de Edad del Bronce de El Estanquillo-Fase II-. 


\section{LAS BASES ARQUEOLÓGICAS PARA LA INTERPRETACIÓN HISTÓRICA DE LA EDAD DEL BRONCE}

Para la consideración de los conjuntos materiales adscritos a la Prehistoria Reciente hemos tenido en cuenta una serie de criterios básicos para la atribución cultural: geomorfológicos, relacionados con la ubicación de los asentamientos y su estratificación y tecnológicos (tipología de la cultura material).

Los criterios geomorfológicos son estratigráficos, basados inicialmente en la secuencia arqueológica del corte 1 de El Estanquillo (Ramos, 1990, 1991, 1993), que ha sido contrastada en toda la ladera sur del Cerro de los Mártires, así como en las laderas y pendientes fundamentalmente de la zona este de San Fernando, que linda al Caño de Sancti Petri (Borja, 1992).

Se comprueba de este modo una sucesión estratigráfica completa de tierras rojas, finas, sueltas, adscritas al Neolítico. Como consecuencia de la fuerte erosión que ha sufrido la zona y por las intensas actividades antrópicas a veces aparecen en superficie, sobre todo en las estribaciones del Cerro de los Mártires, en el escarpado actual de El Estanquillo, junto a unas baterias militares, o en las huertas ubicadas en las inmediaciones del Muelle de Gallineras. También se han documentado estratigráficamente en un perfil de la Avenida de la Constitución.

A ellas se superponen tierras beiges-amarillentas espesas, más claras que las anteriores, adscritas en El Estanquillo, estrato III, fase II al Bronce Pleno. Esto se comprueba y ratifica en los depósitos de cabañas de La Marquina B, con cerámicas relacionadas con el Bronce Pleno, y en un silo -La Marquina A-.

De las 26 localizaciones prehistóricas que tenemos controladas por el momento en San Fernando (RAMOS et al. , 1993), analizando la relación de los yacimientos en su estratificación natural y tras el estudio de sus elementos de cultura material, 9 tienen una ocupación de la Edad del Bronce. Se trata de: El Estanquillo-Fase II-, Camposoto, La Marquina A, La Marquina B, La Marquina C, Pago de la Zorrera, Huerta de Suraña A, Huerta de Suraña B y Edificio Berenguer (Figuras 1, 2).

Tres enclaves de ellos tienen dos ocupaciones, Neolítica y de Edad del Bronce: Camposoto, La Marquina C y Pago de la Zorrera. Tienen ocupación monofásica, estratificada en depósitos arqueológicos de Edad del Bronce: El Estanquillo-Fase II-, La Marquina A y La Marquina B. Corresponden a yacimientos de superficie en depósitos de suelos pardos sobre arenas, Huerta de Suraña A, en posición secundaria y Huerta de Suraña B sobre el mismo depósito, pero estando in situ, en posición primaria'.

\section{ANÁLISIS MORFOLÓGICO Y TIPOLÓGICO DE LOS PRODUCTOS ARQUEOLÓGICOS}

\subsection{Productos cerámicos}

El estudio que presentamos se basa en la documentación de los complejos cerámicos de: El EstanquilloFase II-, La Marquina A y La Marquina B. Consideramos productos cerámicos bien contextualizados, procedentes de lugares de hábitat, estratificados y organizados funcionalmente en su distribución espacial en El Estanquillo-Fase II- (Ramos, 1993) en diversas áreas, predominando en dos estructuras de consumo,

1. La numeración de yacimientos de las Figuras 1 y 2 corresponde a la general de yacimientos que controlamos en San Fernando (Ramos et Al. , 1993: 363). Limitaciones de espacio no permiten un estudio detenido de cada yacimiento. Lo remitimos a una próxima monografía que preparamos, donde detallamos para cada yacimiento un enmarque geográfico, prestando atención a su enclave, rasgos topográficos, altitud, área de dispersión, carácter primario o circunstancias postdeposicionales, con las correspondientes exposiciones cuantitativas de las industrias líticas y cerámicas. 
factor que condicionará la propia ordenación tipológica (Figura 3). Las cerámicas de La Marquina B proceden también de una cabaña, constituyendo formas típicas de consumo o de producción para el consumo. Por su parte el cuenco carenado de La Marquina A se documentó en una estructura de almacenaje-silo.

Por tanto, a pesar de ser complejos cerámicos reducidos, queremos destacar la garantía de estratificación de los 3 enclaves, con gran homogeneidad tecnológica y sincrónica.

La ordenación tipológica, la deducimos por rasgos morfológicos y tipométricos, para quedar valorados en «productos» funcionales, dado que han podido ser analizados en cuanto artefactos contextualizados, en el seno fundamentalmente de actividades domésticas.

Diferenciamos los siguientes grupos tipológicos ${ }^{2}$ (Figura 5):

A. Cerámicas lisas

1. Cuencos.

1.A. Escudillas.

1.B. De casquete esférico y semiesférico.

1.C. De borde entrante.

1.D. Parabólicos.

1.E. Grandes cuencos o cazuelas.

1.F. Grandes cuencos de borde entrante.

2. Ollas.

2.A. Ollas con borde indicado entrante.

2.B. Ollitas con borde saliente.

3. Vasos carenados.

4. Vasos bicónicos y lenticulares.

5. Vasos groseros con perfil en «S» o de paredes verticales.

6. Orzas.

7. Varios.

7.A. Queseras,

7.B. Fusayolas.

B. Cerámicas decoradas.

8. Cerámicas tipo Cogotas.

\subsection{Productos líticos}

Por razones de espacio expondremos un muy sucinto panorama técnico y tipológico de las industrias líticas de la Edad del Bronce de San Fernando.

La materia prima es prioritariamente sílex, que se documenta en forma de cantos y guijarros, de pequeñas dimensiones, en los entornos de San Fernando.Morfológicamente los productos de talla y los artefactos elaborados de Edad del Bronce, difieren notoriamente de los de ocupaciones anteriores por el predominio de los colores negros y grises, sin estar prácticamente patinados. Además del sílex es significativa la presencia de cuarcitas talladas, tanto en los conjuntos de superficie, como en El EstanquilloFase II-.

2. Por las mismas razones indicadas en la nota (1), no podemos desarrollar los rasgos morfométricos y técnicos de las cerámicas (calidades, texturas, pastas, coloraciones, fuegos, cocciones), que definen y precisan la mencionada tipología. 


\section{CUADRO 1. INVENTARIOS PORCENTUALES DE LAS INDUSTRIAS LÍTICAS}

\section{A. CÓMPUTO GENERAL DE RESTOS DE TALLA Y ÚTILES}

$\begin{array}{ccccccccc} & \mathrm{N} & \mathrm{L} & \mathrm{E} & \mathrm{D} & \mathrm{P} & \mathrm{TRT} & \mathrm{U} & \mathrm{T} \\ 1 . & 29 & 211 & 11 & 6 & 1 & 258 & 20 & 278 \\ & 10^{\prime} 43 & 75^{\prime} 90 & 3^{\prime} 96 & 2^{\prime} 16 & 0^{\prime} 36 & 92^{\prime} 81 & 7^{\prime} 19 & 100 \\ 2 . & 25 & 136 & 11 & 24 & 1 & 197 & 37 & 235 \\ & 10^{\prime} 69 & 58^{\prime} 12 & 4^{\prime} 70 & 10^{\prime} 25 & 0^{\prime} 43 & 84^{\prime} 19 & 15^{\prime} 81 & 100 \\ 3 . & 5 & 13 & 4 & 6 & - & 28 & 3 & 31 \\ & 16^{\prime} 13 & 41^{\prime} 93 & 12^{\prime} 90 & 19^{\prime} 38 & - & 90^{\prime} 34 & 9^{\prime} 69 & 100\end{array}$

$\mathrm{N}=$ Núcleos, $\mathrm{L}=$ Lascas, $\mathrm{E}=$ Esquirlas, $\mathrm{D}=$ Desechos, $\mathrm{P}=$ Plaquetas,

TRT $=$ Total Restos de Talla, $\mathrm{U}=$ Utiles, $\mathrm{T}=$ Total.

\section{B. CÓMPUTO GENERAL DE NÚCLEOS}

$\begin{array}{cccccccccccc} & \text { SC } & \text { IT } & \text { LE } & \text { D } & \text { GL } & 1 \text { PG } & 2 \text { PG } & \text { HO } & \text { SL } & \text { DI } & \text { T } \\ \text { 1. } & - & 2 & 4 & 5 & 2 & 7 & 3 & - & 2 & 4 & 29 \\ & - & 6^{\prime} 90 & 13^{\prime} 79 & 17^{\prime} 24 & 6^{\prime} 90 & 24^{\prime} 14 & 10^{\prime} 34 & - & 6^{\prime} 90 & 13^{\prime} 79 & 100 \\ \text { 2. } & 1 & - & 13 & - & 8 & - & - & 1 & 1 & 1 & 25 \\ & 4^{\prime} 00 & - & 52^{\prime} 00 & - & 32^{\prime} 00 & - & - & 4^{\prime} 00 & 4^{\prime} 00 & 4^{\prime} 00 & 100 \\ 3 . & 1 & - & 2 & - & - & - & - & - & 2 & - & 5 \\ & 20^{\prime} 00 & - & 40^{\prime} 00 & - & - & - & - & - & 40^{\prime} 00 & - & 100\end{array}$

$\mathrm{SC}=$ Sobre cantos, $\mathrm{IT}=$ Del inicio de la talla, $\mathrm{LE}=$ Levallois, $\mathrm{D}=$ Discoides, $\mathrm{GL}=$ Globulosos, $1 \mathrm{PG}=\mathrm{Con}$ un plano de golpeo, $2 \mathrm{PG}=$ Con dos planos de golpeo, $\mathrm{HO}=\mathrm{Para}$ hojas, $\mathrm{SL}=$ Sobre lascas, $\mathrm{D}=$ Diversos, $\mathrm{T}=\mathrm{T}$ otal.

\section{CÓMPUTO GENERAL DE LASCAS}

$\begin{array}{ccccccccc} & \text { DE } & \text { SD } & \text { IN } & \text { LE } & \text { CR } & \text { HO } & \text { SO } & \text { T } \\ \text { 1. } & 7 & 11 & 174 & 18 & 1 & - & - & 211 \\ & 3^{\prime} 32 & 5^{\prime} 21 & 82^{\prime} 46 & 8^{\prime} 54 & 0^{\prime} 47 & - & - & 100 \\ 2 . & 4 & 15 & 97 & 15 & 2 & 2 & 1 & 136 \\ & 2^{\prime} 94 & 11^{\prime} 03 & 71^{\prime} 32 & 11^{\prime} 03 & 1^{\prime} 47 & 1^{\prime} 47 & 0^{\prime} 74 & 100 \\ 3 . & - & - & 11 & 2 & - & - & - & 13 \\ & - & - & 84^{\prime} 61 & 15^{\prime} 39 & - & - & - & 100\end{array}$

$\mathrm{DE}=\mathrm{De}$ descortezado, $\mathrm{SD}=$ De semidescortezado, $\mathrm{IN}=$ Internas, $\mathrm{LE}=$ Levallois, $\mathrm{CR}=\mathrm{De}$ crestas, $\mathrm{HO}=\mathrm{Hojas}, \mathrm{SO}=$ Sobrepasadas, $\mathrm{T}=$ Total.

\section{CÓMPUTO GENERAL DE ÚTILES}

$\begin{array}{ccccccccccccc} & \mathrm{R} & \mathrm{P} & \mathrm{M} & \mathrm{D} & \mathrm{T} & \mathrm{S} & \mathrm{A} & \mathrm{RU} & \mathrm{AS} & \mathrm{EH} & \mathrm{DI} & \mathrm{T} \\ 1 . & 1 & 1 & 2 & - & 3 & 2 & - & - & - & 11 & - & 20 \\ & 5^{\prime} 00 & 5^{\prime} 00 & 100^{\prime} 00 & - & 15^{\prime} 00 & 10^{\prime} 00 & - & - & - & 55^{\prime} 00 & - & 100 \\ 2 . & - & - & 10 & 2 & 1 & - & - & 4 & - & 1 & 20 & 38 \\ & - & - & 27^{\prime} 03 & 5^{\prime} 41 & 2^{\prime} 70 & - & - & 10^{\prime} 81 & - & 2^{\prime} 70 & 51^{\prime} 35 & 100 \\ 3 . & - & - & 1 & - & - & - & 1 & - & 1 & - & - & 3 \\ & - & - & 33^{\prime} 33 & - & - & - & 33^{\prime} 33 & - & 33^{\prime} 33 & - & - & 100\end{array}$

$\mathrm{R}=$ Raspadores, $\mathrm{P}=$ Perforadores, $\mathrm{M}=$ Muescas, $\mathrm{D}=$ Denticulados, $\mathrm{T}=$ Truncaduras, $\mathrm{S}=$ Retoques simples, $\mathrm{A}=$ Retoques Abruptos, $\mathrm{AS}=$ Lascas astilladas, $\mathrm{EH}=$ Elementos de hoz, $\mathrm{DI}=$ Diversos. $\mathrm{T}=$ Total.

1.= EL ESTANQUILLO-FASE II-, 2.= HUERTA DE SURAÑA A, 3.= HUERTA DE SURAÑA B. 


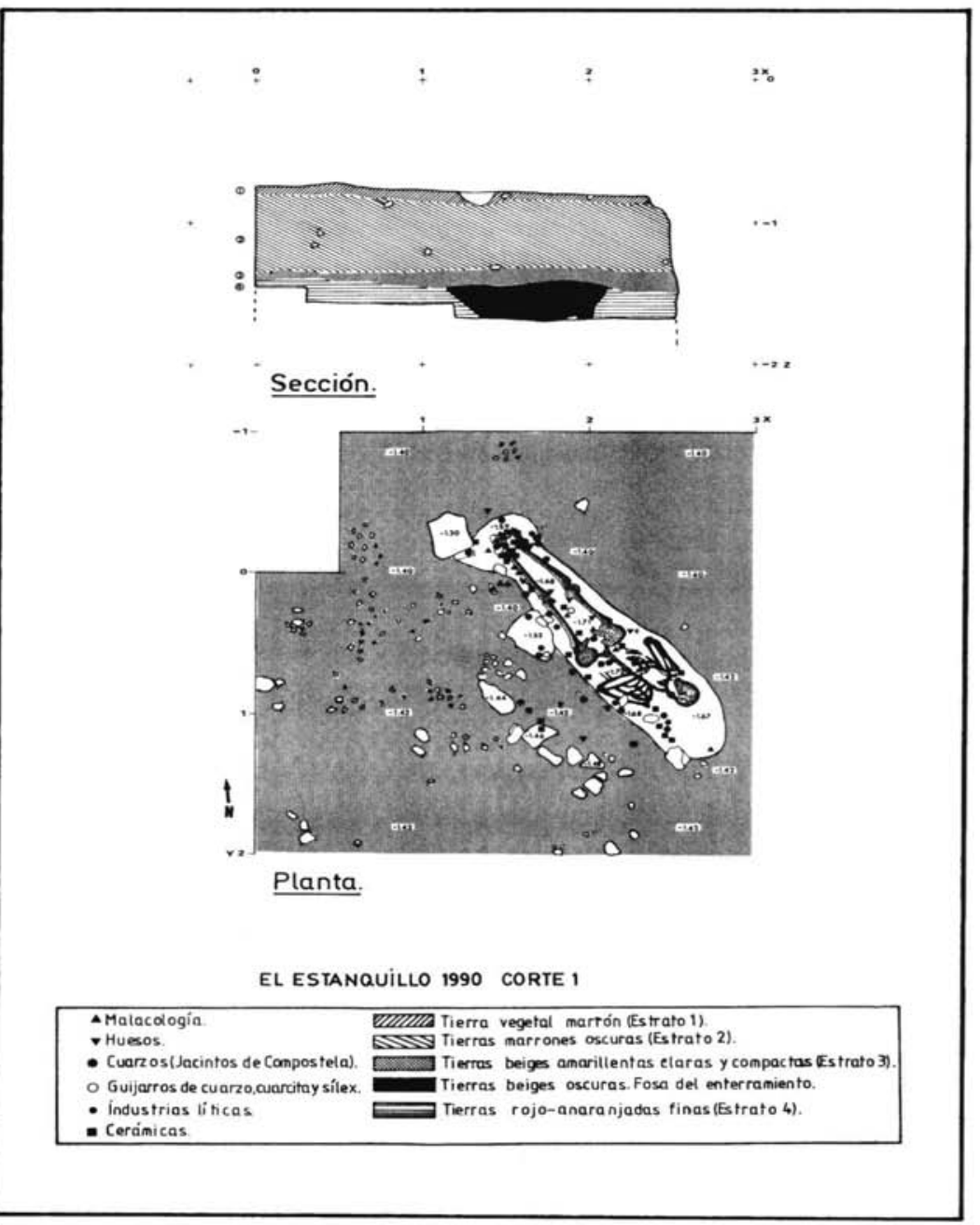

FIGURA 4. Enterramiento de Edad del Bronce de El Estanquillo. 
El cómputo general de restos de talla y útiles (Cuadro 1A) pone en evidencia el manifiesto predominio de los restos de talla. Funcionalmente esto se comprueba de forma clara en El Estanquillo, por los productos de un área de taller, formado por 45 lascas, 2 esquirlas y 1 desecho (Ramos, 1993). En el total de la Fase II, los restos de talla alcanzan el 92'81\%, comprobándose los diferentes elementos de la «cadena operativa» del desbaste «in situ», núcleos (10’43\%), lascas (75'90\%) así como esquirlas (3’96 \%), desechos $\left(2^{\prime} 15 \%\right)$ y plaquetas de avivamiento $\left(0^{\prime} 36 \%\right)$. Este predominio de restos de talla se comprueba también en Huerta de Suraña A, con 84'19 \% y Huerta de Suraña B, 90’34 \%.

Dado el reducido espacio físico de aparición de los objetos líticos en Huerta de Suraña B, 10 x 10 mts. , así como la propia configuración de los restos de talla, lo valoramos como un característico lugar de taller, al estilo del taller doméstico de El Estanquillo, en relación con las actividades de hábitat y productivas que se realizaban en los entornos de Huerta de Suraña.

Los núcleos nos demuestran que aún no se observa un decaimiento significativo de las técnicas de talla (Cuadro 1B), con gran variedad de estilos y sistemas de desbaste (10 tipos documentados). Son característicos, los realizados sobre cantos, y del inicio de la talla, levallois, e incluso centrípetos de buena calidad; no se han documentado núcleos prismáticos y sólo un buen ejemplar para hojas; con planos de golpeo preparados, sobre lascas y diversos.

Tecnológicamente estos núcleos conllevan un desbaste uniforme de lascas con talones corticales y lisos, con un predominio de los tipos pequeños y medianos, y mayor producción de lascas que de láminas.

Entre las lascas comprobamos (Cuadro 1C) un predominio generalizado de las de talla interna, con escasa presencia de lascas de talla levallois y casi desaparición de las hojas. El desbaste es pues mucho más funcional y está en estrecha relación al producto a obtener.

En cuanto a los útiles (Figura 6) representan una interesante aproximación a la tecnología de producción, pues nos marcan dos tendencias tecnológicas claves, para la comprensión de la estructura económica de esta formación social. Por un lado, los artefactos vinculados con la agricultura y por otro con la recolección de malacofauna (Cuadro 1D).

Los útiles de tradición paleolítica son escasos, corresponden a un raspador y a un perforador, en El Estanquillo, reflejo de actividades domésticas. El perforador se documentó en el ajuar del enterramiento, y se vincula junto a los cantos tallados -incluidos entre los diversos- a las actividades de marisqueo. Las muescas conforman el $2^{\circ}$ mejor tipo documentado, tras los cantos. Están presentes denticulados y truncaduras. Estos tres últimos tipos se vinculan con el proceso de elaboración de elementos de hoz, como pudo demostrar la relación de la hoz, con el pequeño taller de El Estanquillo (Ramos, 1993).También se documentan lascas retocadas, con retoques simples, abruptos y de uso, así como lascas astilladas. Los elementos de hoz juegan un papel importante como artefactos de producción. Es a destacar la localización de una hoz completa formada por 9 elementos en el área de producción de El Estanquillo (Figura 6), asociada a un núcleo discoide, y 2 truncaduras, que interpretamos como una hoz en proceso de reparación, obteniendo las lascas de un pequeño taller adyacente (Ramos, 1993). En cuanto a los cantos, son de talla unifacial y bifacial, vinculados junto a perforadores y lascas brutas, a actividades de marisqueo, conforman un fenómeno de cierto alcance, dado el importante papel económico que ésta actividad tiene para las comunidades de Edad del Bronce de este territorio. 


\section{VALORACIÓN FUNCIONAL Y ECONÓMICA DE LOS PRODUCTOS ARQUEOLÓGICOS}

\subsection{Productos cerámicos}

Los conjuntos cerámicos procedentes de El Estanquillo-Fase II-, La Marquina A y La Marquina B, permiten una adscripción amplia en contextos históricos de la Edad del Bronce. Son formas características de mediados del II $^{\circ}$ milenio a.C. del Occidente de Andalucía y en general del S.O. peninsular.

En el trabajo monográfico de El Estanquillo (Ramos, 1993) realizamos un enmarque regional amplio de contrastación de las formas documentadas con yacimientos que han permitido una ordenación secuencial para los inicios y momentos plenos de la Edad del Bronce del Occidente de Andalucía. Sólo cabe mencionar el contexto de sintonía histórica con las fases II y III de El Berrueco (Medina Sidonia) (Escacena y de Frutos, 1981-1982, 1985), con el estrato III, de la Calle Alcazaba de Lebrija (Caro, A. , 1989, 1991; Caro et Al. , 1986), con la fase I, estratos XIV y XV de Mesa de Setefilla (Lora del Río, Sevilla) (Aubet y Serna, 1981; Aubet, et Al. , 1983; Serna et Al. , 1984) y en un modo amplio con los poblados de la cuenca del río Odiel (Huelva) (Nocete et Al. ,1993); o El Trastejón (Zufre, Huelva) (Hurtado, 1988, 1989), así como con el denominado Horizonte de Atalaia, del S.O. de Portugal (Schubart, 1975).

Ahora bien, creemos que sólo el enmarque territorial, definido diacrónica y espacialmente podrá aportarnos criterios válidos de contrastación (paralelos, semejanzas, definición de centros de producción locales y distribución de productos ajenos por medio del intercambio), para los conjuntos cerámicos.

$\mathrm{Al}$ no estar aún bien definido el territorio de esta formación social no podemos más que presentar los contextos cerámicos y realizar un modelo de interpretación de tipología funcional (Figura 5) en entornos de la periferia, de asentamientos domésticos, que reflejan un hábitat permanente, para actividades productivas y recolectoras.

Conviene destacar el predominio cuantitativo de formas de consumo (Formas 1A, 1B, 1C, 1D, 1E, 3), caracterizadas por la gran variedad del grupo de cuencos. Predominan cuantitativamente los de borde entrante, sobre los de casquete esférico-semiesférico y las cazuelas, siendo escasa la presencia de escudillas. Los cuencos dominan en El Estanquillo-Fase II-, asociados a un área de consumo, junto a hogares y restos de una comida(Figura 3). Son también significativos entre los conjuntos descontextualizados de El Estanquillo, lo que demuestra el predominio de estas actividades en otras zonas del asentamiento, no excavadas y en parte ya destruidas.

Hemos constatado también, formas de producción para el consumo. Este grupo recoge por un lado vasos bicónicos y lenticulares (Forma 4), así como vasos con perfil en «S» y de paredes verticales (Forma 5), documentados en El Estanquillo-Fase II- en el interior de hogares, donde se calentaban y preparaban alimentos en el fuego, para un posterior consumo. También se incluyen en este grupo, fragmentos de quesera-colador (Forma 7), como verdadero artefacto de elaboración-transformación de alimentos.

Se documentan formas de almacenaje, por un lado, los grandes cuencos de borde entrante (Forma 1F), algunas ollas (Forma 2) y las orzas (Forma 6). Es a destacar la escasa representación cuantitativa de este grupo. Esto indica claramente que estamos ante comunidades productoras y depredadoras, que no conservan excedentes, y que la producción cerealista debe ir orientada hacia áreas de centros nucleares. De hecho los productos documentados en el silo de La Marquina A, son malacológicos, reflejando un pequeño almacenaje para el consumo doméstico.

Las formas de prestigio, o que tienen un carácter votivo son escasas, se reducen al vaso carenado del ajuar del enterramiento de El Estanquillo-Fase II- (Forma 3), englobando algunos vasos carenados. Incluimos en este grupo al cuenco con decoración de estilo Cogotas -hasta la comprobación de su 


\begin{tabular}{|c|c|c|c|c|c|}
\hline & \multicolumn{5}{|c|}{$\begin{array}{l}\text { FUNCIONALIDAD DE LAS CERAMICAS CONTEXTUALIZADAS } \\
\text { DE LA EDAD DEL BRONCE DE SAN FERNANDO. }\end{array}$} \\
\hline FORMAS & CONSUMO & $\begin{array}{l}\text { PRODUCCION } \\
\text { PARA CONSUMO }\end{array}$ & DE PRESTIGIO & ALMACENAJE & $\begin{array}{l}\text { ACTIVIDAD } \\
\text { TEXTIL }\end{array}$ \\
\hline $1 \mathrm{~A}$ & 4 & & & & \\
\hline $1 \mathrm{~B}$ & & & & & \\
\hline $1 \mathrm{c}$ & & & & & \\
\hline 10 & & & & & \\
\hline $1 \mathrm{E}$ & & & & & \\
\hline $1 \mathrm{~F}$ & & & & & \\
\hline 2 & & & & & \\
\hline 3 & 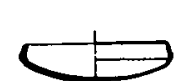 & & 0 & & \\
\hline & & & & & \\
\hline 8 & & & & & \\
\hline 6 & & & & & \\
\hline 7 & & $\because \because a$ & & & $\circ 8$ \\
\hline 8 & & & $5 \theta$ & & \\
\hline
\end{tabular}

FIGURA 5. Tabla de formas e hipótesis de funcionalidad de las cerámicas de Edad del Bronce de San Fernando: EI Estanquillo-Fase II- y La Marquina B. 
procedencia por el análisis de pastas-, en una valoración como objeto decorativo, a pesar de su ubicación en un ambiente doméstico de la cabaña de La Marquina $\mathrm{B}^{3}$.

Actividades textiles completan el panorama, con las fusayolas de El Estanquillo.

\subsection{Tecnología lítica}

La tecnología lítica como expresión de las herramientas que posibilitan actividades productivas básicas, son fundamentales para la comprensión de la estructura económica de las comunidades prehistóricas. La tecnología lítica tallada en la Edad del Bronce del territorio estudiado de San Fernando, nos informa fundamentalmente de 4 tipos de actividades:

\section{a) Tecnología lítica vinculada con actividades de producción para el sustento básico}

Constituye el grupo tecnológico más significativo. Cuenta con los elementos de hoz y los útiles en proceso de elaboración, donde se pueden incluir, muescas, denticulados y truncaduras. Son elementos básicos para la siega y reflejan la importancia de la agricultura de secano de cereales, en el desarrollo de las fuerzas productivas de estas comunidades de periferia costera, pero con buenas tierras adyacentes.

De la hoz en reparación de El Estanquillo-Fase II- (Figura 6) se obtienen interesantes datos de índole etnográfica (Ramos , 1990-1991). La presencia del lustre del cereal indica que ya había sido intensamente empleada, antes de ser llevada al poblado. Informa de aspectos tecnológicos sustantivos: forma de las hoces prehistóricas en cuanto utensilios compuestos, posicionamiento de los propios elementos en la hoz, sistemas de engarce de las truncaduras y dorsos abatidos, colocación espacial diferenciada de elementos triangulares y rectangulares en la hoz. Además documenta procesos de reparación de tecnología básica de producción. Nos muestra que se acondicionaban las hoces en los asentamientos, asociada dicha actividad con pequeños talleres domésticos (Ramos, 1993).

\section{b) Tecnología lítica relacionada con actividades de depredación}

Se incluye a perforadores y grupo de cantos tallados, de talla unifacial y bifacial, así como probablemente a lascas internas y levallois con retoques de uso y melladuras. Se vinculan a las actividades de marisqueo y recolección de especies malacológicas, siendo estos artefactos utilizados para despegar de las rocas las especies, así como para la apertura de las mismas. Estas actividades depredadoras son fundamentales para el sustento de esta comunidad, como demuestra la Fase II de El Estanquillo, generan incluso pequeños almacenajes como el silo de La Marquina A y pensamos que llegaron a entrar incluso en un circuito de distribución hacia tierras del interior.

\section{c) Tecnología lítica asociada con actividades domésticas}

Se incluyen aquí raspadores, algunas muescas, lascas retocadas, vinculadas con trabajos domésticos, como cortar, acondicionamiento de pieles... Son el reflejo de perduraciones clásicas de la Prehistoria, que en la Edad del Bronce han perdido ya un valor sustancioso, contrastado con el papel de estas actividades en el Paleolítico Superior, Epipaleolítico y Neolítico. Su débil representación marca la tendencia más funcional-pragmática de la tecnología de estos momentos, en clara correlación con la estructura económica de estas comunidades.

3. El fenómeno Cogotas I en Andalucía se inserta cronológicamente en la problemática de la definición del Bronce Tardío. Este vendría a definir unas manifestaciones culturales diferenciadas, situadas entre el Bronce Medio y el Bronce Final, identificable con un horizonte post-argárico sólo en el sudeste, pero en general puede vincularse como estilo decorativo cerámico con formaciones de sociedades del Bronce Pleno de otras áreas del Mediodía (Arteaga, 1981). 


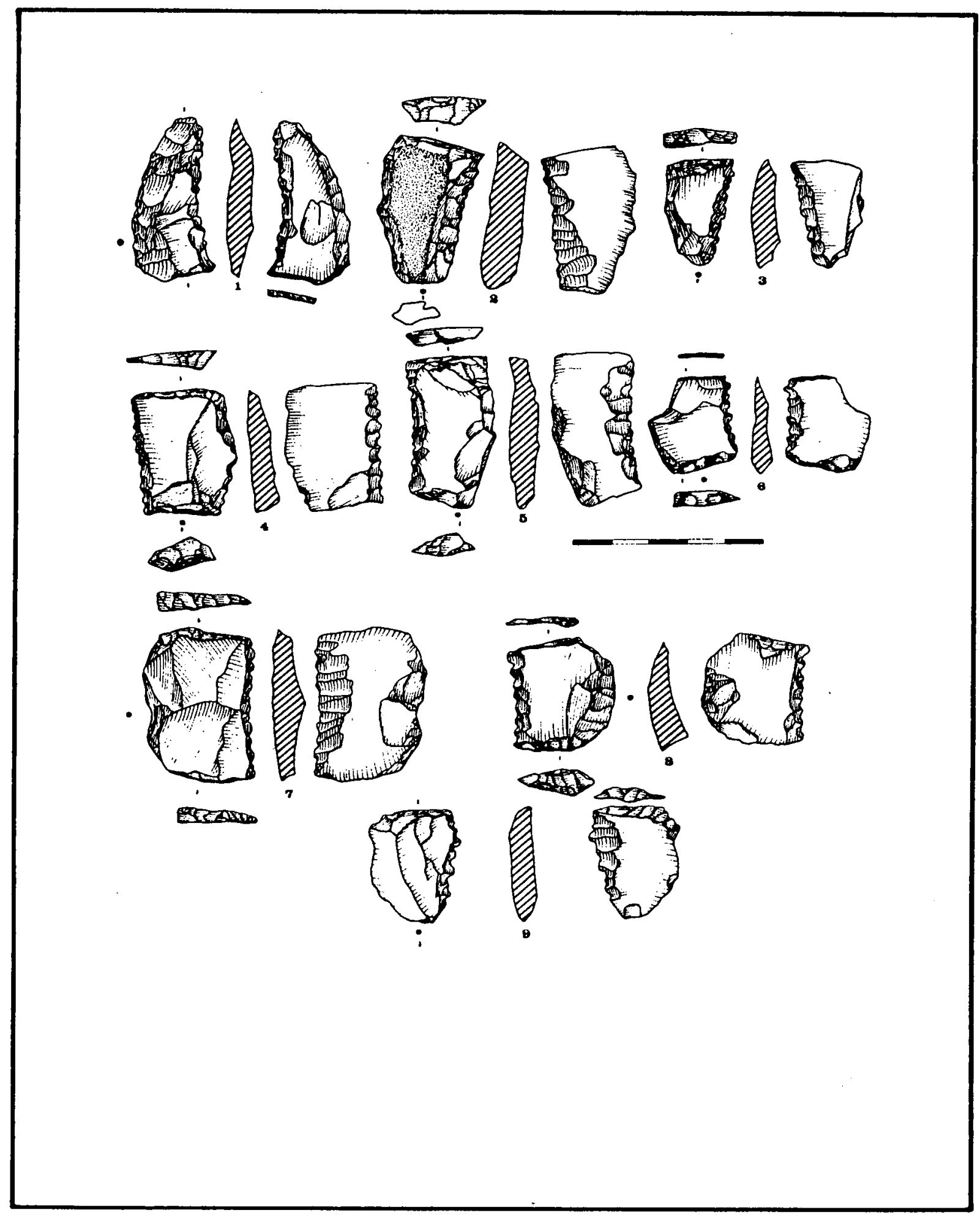

FIGURA 6. El Estanquillo-Fase II- Elementos de hoz. 


\section{d) Tecnología lítica asociada a actividades votivas, de ritual o prestigio}

Es un elemento del mundo superestructural-ideológico, que ha debido ser más significativo que el recogido por la bibliografía especializada. Los componentes líticos formaron también parte de los ajuares de enterramientos individuales de Edad del Bronce. En El Estanquillo-Fase II- en la propia fosa de inhumación, asociados al enterramiento se documentaron 24 objetos líticos tallados: 2 esquirlas, 1 desecho, 3 lascas de semidescortezado, 14 lascas internas, 3 lascas levallois y 1 perforador. Además se depositaron 79 guijarros de cuarzo (Ramos, 1993), distribuidos todos estos productos en la fosa de inhumación (Figura 4). La presencia de desechos, esquirlas, lascas de talla externa e interna, nos habla de una especie de talla ritual sobre el enterramiento una vez depositado el cadáver en la fosa. Por su parte el perforador debió contar con un mango, que se encontraba adyacente a la mano izquierda.

\section{IMPLICACIONES ECONÓMICAS, SOCIALES Y POLÍTICAS DE LA EDAD DEL BRONCE EN SAN FERNANDO}

\section{7.a. Delimitación territorial}

La delimitación territorial en la que se sitúa el poblamiento de Edad del Bronce constatado en San Fernando, va a ser clave fundamental para la comprensión de su proceso histórico. Aún no tenemos definido dicho territorio político del $\mathrm{II}^{\circ}$ milenio a.C., dado que hemos comenzado en 1992 el mencionado proyecto de investigación, pero intuimos una ubicación interior del centro nuclear de dicha formación económica y social, en torno a Medina Sidonia, en buenas y fértiles campiñas, controlando un espacio amplio, de la desembocadura del río Guadalete, Bahía de Cádiz, Banda Atlántica y campiñas interiores de Chiclana de la Frontera, Medina Sidonia, Benalup y buena parte de Jerez de la Frontera.

Factores estratégicos, de ubicación, de control visual, de captación del mejor territorio de producción, inciden a señalar el área nuclear en los entornos de Medina Sidonia. Allí se enclavan poblados como, Cerro El Berrueco (Escacena y de Frutos, 1985) o Las Mesas (Chiclana), que ejercen un control del territorio jerarquizado, en la vía de acceso del río Iro, hacia la zona periférica occidental de la Bahía de Cádiz.

La excavación en El Estanquillo, las prospecciones arqueológicas sistemáticas en San Fernando y el conocimiento que vamos teniendo de la Prehistoria de la Bahía de Cádiz (Ramos, en prensa; Gutierrez, et Al. , en prensa) nos permiten considerar la hipótesis de que en la isla de San Fernando, a mediados del II $^{\circ}$ milenio a.C. se establece un modelo de organización económico-social, que representa una «periferia» insular, con clara dependencia territorial, económica y socio-política del mencionado centro nuclear.

\section{7.b. Modelo de asentamientos}

Hemos comprobado un modelo de asentamiento homogéneo, sin estructuras defensivas, que ocupan reducidas dimensiones, en la zona sur de San Fernando, ubicados en suelos que fisiográficamente son de llanura y suave pendiente, de gran calidad para los cultivos de secano (García del Barrio, 1988), orientados a las marismas y Caño de Sancti Petri. Hemos documentado 9 pequeños enclaves: El Estanquillo, Camposoto, La Marquina A, La Marquina B, La Marquina C, Pago de la Zorrera, Huerta de Suraña A, Huerta de Suraña B y Edificio Berenguer (Figuras 1,2).

Visualmente todos son dominados desde Camposoto, que constituye un hábitat con clara función estratégica.Todos estos asentamientos están orientados hacia el S.E., observando visualmente el área nuclear del entorno de Medina Sidonia, desde el Cerro de los Mártires, ocupando un área de unos 2.500 mts., en sentido N.-S. por 1.000 mts. en sentido E.-O. . 
Hemos realizado una preliminar ordenación funcional de dichos enclaves en relación a estructuras y productos arqueológicos:

- Cabañas: La Marquina B.

- Silo: La Marquina A.

- Talleres líticos: La Marquina C y Huerta de Suraña B.

- Función estratégica de hábitat, con elementos de producción: Camposoto.

- Actividad doméstica polifuncional:El Estanquillo-Fase II-.

La excavación arqueológica de urgencia en El Estanquillo (Ramos, 1991, 1993) permitió documentar un abandono in situ de la Fase II, habiendo quedado todos los productos arqueológicos en su deposicióndisposición, tras el abandono por la comunidad de Edad del Bronce (Figura 3). Procesos coluviales aportaron material desde las cercanas laderas del Cerro de los Mártires y propiciaron una rápida colmatación de este nivel ${ }^{4}$. Esto permitió la conservación de estructuras arqueológicas, que han sido importantes testimonios de áreas de actividad: área de consumo (dos hogares y restos de los desperdicios de una comida), área de producción (molino con cazoletas, pequeño taller lítico, una hoz) y área de enterramiento, ubicada bajoe el piso de ocupación del asentamiento (Figura 4).

\section{7.c. Hipótesis de trabajo. Análisis de la estructura económica de la formación social del $\mathrm{II}^{\circ}$ milenio a.C. en el territorio de campiñas y litoral Atlántico de Cádiz}

Las explicaciones conducentes a la delimitación espacial y territorial de la formación económica y social que se articuló en las campiñas centrales y litorales de Cádiz a mediados del $\mathrm{II}^{\circ}$ milenio a.C. tienden a la definición de un centro nuclear y una periferia.

La exposición de la estructura económica de la periferia, muestra una dependencia política de ésta, situada en los entornos de San Fernando, Cádiz, Bahía de Cádiz, y parte del litoral Atlántico, respecto a un centro coercitivo político.

Somos conscientes de la dificultad que conlleva la explicación de un modelo político económico, con explotación de clases, desde la periferia. Pero las circunstancias de la investigación nos han conducido a ésta coyuntura y situación.

El estudio de los yacimientos de Edad del Bronce de San Fernando nos indican que estos asentamientos reflejan un proceso de producción significativo, con notorias evidencias de consumo -nivel de reproducción doméstica del desarrollo de las fuerzas productivas-, con práctica ausencia de actividades de almacenaje -nivel de concentración de excedentes para una redistribución-. Se ha podido confirmar que los componentes que reflejan una actividad de almacenaje, documentada por grandes cuencos entrantes, orzas, ollas de El Estanquillo y silo de La Marquina A, indican elementos de almacenaje doméstico para el autoconsumo.

Así, los excedentes generados por la producción agrícola, se debieron conducir hacia otras áreas, que a nivel de hipótesis creemos deben situarse en torno al centro nuclear que en sentido amplio ubicamos en el entorno de El Berrueco-Las Mesas-Medina Sidonia. Igualmente creemos que dada la importancia alcanzada por el consumo de malacofauna, ha debido incidir en una cierta distribución hacia el interior de productos malacológicos.

4. «La conservation des vestiges d'habitat dépend en premier lieu de la rapidité avec laquelle ils ont été enfouis. Plus la durée pendant laquelle les vestiges sont restés sans protection a été longue, plus leurs conditions de conservation sont mauvais» (Bosinsski, 1990: 18). 
Por tanto, el desarrollo de las fuerzas productivas en San Fernando se nos documenta por el papel predominante de la agricultura, evidenciada por la ubicación de los enclaves adyacentes a los buenos y productivos suelos de tipo Rincones, muy aptos para el cultivo del cereal, y confirmado por la tecnología especializada en elementos de hoz, con importante comprobación en El Estanquillo de actividades de reparación de piezas para una hoz, así como actividades de molienda (molino y moletas).

Evidencias de actividades de desforestación y trabajo con la madera se comprueban por los componentes pulimentados documentados (azuelas, escoplos) que creemos de producción local, a partir del afloramiento de ofitas del Trías Superior del Cerro de los Mártires (Zazo et Al., 1987).

El proceso de desforestación, nos pone en relación con procesos de ganar terreno a la vegetación natural ${ }^{5}$, lo que se confirma por el análisis geomorfológico (Borja, 1992, Borja et Al. , en prensa, Borja y Ramos, en prensa a, b), al comprobar la existencia de procesos de arroyada, con lo cual la vegetación estaba ciertamente degradada.

La producción agrícola, aparte de la importancia que conlleva, para el autoabastecimiento local, generó un aporte sustancial hacia el área del centro nuclear. Ello debió generar en ésta, un importante fenómeno de concentración de excedentes agrícolas. (La gran presencia de elementos cerámicos de almacenaje avala esta hipótesis en poblados del área nuclear, caso de La Mesa).

El factor de la ganadería y domesticación también fue fundamental, documentadas en el importante papel que la fauna ocupa en el Estanquillo-Fase II-, fundamentalmente, bovinos y ovinos, en estudio por E. Bernáldez. La propia denominación del topónimo «La Dehesa» habla del papel gandero en esta zona sur de San Fernando, y se confirma por la presencia de queseras, para la transformación-manipulación de alimentos.

El desarrollo de las fuerzas productivas genera una importante serie de actividades de depredación, donde el marisqueo conllevó una aportación sustantiva en la dieta, como lo demuestra la significativa documentación de especies malacológicas. Su importante valor en la vida de estas comunidades se comprueba incluso en el mundo ritual -enterramiento de El Estanquillo (Figura 4)- y hasta en el reducido almacenaje constatado en el silo de La Marquina A. En este sentido creemos que los productos malacológicos también han debido entrar en el circuito de distribución, al igual que los cereales.

La articulación de las relaciones de producción-fuerzas productivas en la periferia, se organiza desde la clara dependencia política del movimiento de excedentes desde la periferia hacia el centro nuclear. Además es importante destacar que las fuerzas productivas como factor de subsistencia, permiten el propio nivel de reproducción doméstica. Dichas relaciones generan y posibilitan el mantenimiento del sistema por un control político-coercitivo. Esta coerción está en la base de las relaciones sociales de producción. Genera por un lado unos parámetros jerarquizados de división en clases, manifestados en una clara división del trabajo. Por un lado artesanal, con surgimiento y desarrollo de especialistas, alejados de la producción directa de bienes de consumo. Así junto a agricultores y ganaderos tenemos significativas actividades domésticas, como reflejo de artesanías especializadas. En la Edad del Bronce de San Fernando, ésto se puede comprobar en:

- Evidencias de artesanía textil en El Estanquillo.

- Reparación de artefactos líticos de producción (vinculación del taller doméstico con la hoz en El Estanquillo-Fase II-), lo que incide en el papel de los artesanos talladores del sílex.

- Los talladores, elaboradores de pulimentados, con evidencias de extracción y manipulación de ofitas locales.

- Molienda-manipulación de cereales en el molino de El Estanquillo.

5. El fenómeno de la desforestación se manifiesta en la Bahía de Cádiz, desde el Neolítico Final, paralelo al desarrollo de las fuerzas productivas y a la instauración de la agricultura (Ramos et Al., 1991, 1992; Valverde, 1991, 1993). 
Actividades domésticas y artesanales pueden llegar a confundirse, ya que también se documentan preparaciones de alimentos para el consumo en hogares y transformación-manipulación de alimentos quesera-colador.

Somos conscientes, en el momento actual de la investigación, de la limitación del registro arqueológico y la necesidad de nuevas prospecciones y excavaciones, tanto en la periferia -litoral-como en el centro nuclear -campiñas del interior-. Esto limita lógicamente los resultados en varios campos, por ejemplo en el contexto de la dificultad de separar la división artesanal del trabajo, respecto a la división sexual del mismo. De hecho muchas de las actividades indicadas, productivas y/o domésticas podrían haber sido realizadas por mujeres.

De cualquier modo, las evidencias expuestas, creemos que generan una clara existencia de división artesanal del trabajo, con la confirmación de la existencia del «no productor de bienes de subsistencia», que realiza tareas domésticas y productivas para el consumo y reparación-transformación tecnológica de los propios medios de producción -taller doméstico-hoz-pulimentados-.

En el propio seno de las relaciones sociales de producción hay evidencias claras de parámetros ideológicos jerarquizados, en la inhumación individual y en su rito, formado por elementos modestos (Ramos, 1993) (Figura 4). Por él podemos ver el fenómeno de la total desintegración de estructuras sociales comunitarias, de índole tribal y la definitiva implantación de los valores «individuales», frente a los «colectivos».

Esta estructura socio-económica de comunidades que trabajan y producen para un centro de dominación, sólo se puede mantener con la presencia de algún aparato coercitivo de índole social, lógicamente de no productores, y basado en un control para el mantenimiento del sistema ${ }^{6}$.

\section{BIBLIOGRAFÍA}

ARTEAGA, O. (1981): «Problemas de la Protohistoria de la Península Ibérica». Boletín de la Asociación Española de Amigos de la Arqueología, 14: 4-16. Madrid.

AUBET, M.E. y SERNA, M.R. (1981): «Una sepultura de la Edad del Bronce en Setefilla (Sevilla)». Trabajos de Prehistoria 38: 225-252. Madrid.

AUBET, M.E. , SERNA, M.R. , ESCACENA, J.L. y RUIZ, M.M. (1983): La Mesa de Setefilla. Lora del Río (Sevilla). Campaña de 1979. Excavaciones Arqueológicas en España, 122. Madrid.

BORJA, F. (1992): Cuaternario Reciente, Holoceno y períodos históricos del SW de Andalucía. Paleogeografía de medios litorales y fluvio-litorales de los últimos 30000 años. Tesis Doctoral. Universidad de Sevilla.

BORJA , F. y RAMOS, J. (En prensa a): «Holoceno Medio y Reciente (<6.000 BP) del litoral atlántico de Cádiz. Paleogeografía y antropización». $2^{a}$ Reunión Nacional de Geoarqueología . Madrid, 1992.

BORJA, F y RAMOS, J. (En prensa b): «Las costas de Cádiz durante los últimos 30.000 años». Cuadernos de Geografía. Universidad de Cádiz. 4.

BORJA , F. , DÍAZ DEL OLMO, F. , RECIO, J.M. y RAMOS, J. (En prensa): «Evolución paleoclimática Tardiglaciar-Holoceno y presencia antrópica en la costa W de Cádiz(España)». Revue de Geomorphologie Dinamique. Estrasburgo.

6. La situación estratégica del emplazamiento de Camposoto la relacionamos con dicha función, pero la gran destrucción de evidencias arqueológicas en dicho yacimiento impide profundizar en la explicación de esta hipótesis. 
BOSINSKI, G. (1990): Homo Sapiens. L'histoire des chasseurs du Paléolithique supérieur en Europe (40.000-10.000 avant J.-C.). Editions Errance. Paris.

CARO, A. (1989): «Consideraciones sobre el Bronce Antiguo y Pleno en el Bajo Guadalquivir». En Tartessos. Arqueología Prtohistórica del Bajo Guadalquivir. M.E. AUBET, Coord.: 85-120. Barcelona.

CARO, A. (1991): Lebrija. La ciudad y su entorno, I (Prehistoria y Protohistoria). Colección Conoce Lebrija $\mathrm{N}^{\circ}$ 2. Lebrija.

CARO, A. , ACOSTA, P. y ESCACENA, J.L. (1986): «Informe sobre la prospección arqueológica con sondeo estratigráfico en el solar de la calle Alcazaba (Lebrija-Sevilla)». Anuario Arqueológico de Andalucía, Vol. II: 168-174. Sevilla.

ESCACENA, J.L. y DE FRUTOS, G. (1981-1982): «Enterramientos de la Edad del Bronce del Cerro del Berrueco (Medina Sidonia, Cádiz)». Pyrenae 17-18: 165-189. Barcelona.

ESCACENA, J.L. y DE FRUTOS, G. (1985): «Estratigrafía de la Edad del Bronce en el Monte Berrueco (Medina Sidonia, Cádiz)». Noticiario Arqueológico Hispánico 24: 9-90. Madrid.

GARCÍA DEL BARRIO, I. (1988): Mapa de suelos de la provincia de Cádiz. Cádiz y San Fernando. E. 1: 50.000 Hoja 4. Junta de Andalucía. Sevilla.

GAVALA, J. (1924): Mapa geológico de la provincia de Cádiz. E. 1: 100.000. I.G.M.E.

GUTIÉRREZ, J.M., RUIZ, J.A. y LÓPEZ, J.J. (En prensa): «El yacimiento arqueológico de Campín Bajo. Su enmarque en el poblamiento de Andalucía Occidental y el Bajo Guadalquivir durante el tránsito del II al I milenio. Una propuesta de interpretación», Revista de Historia de El Puerto 10. El Puerto de Santa María.

HURTADO, V. (1988): «Excavaciones en el yacimiento de el Trastejón (Zufre, Huelva). Primera Campaña, 1988. Informe Preliminar». Anuario Arqueológico de Andalucía, Vol. II:158-164. Sevilla.

HURTADO, V. (1989): «El yacimiento de el Trastejón (Zufre, Huelva). Estudios de materiales. Informe de la campaña 1989». Anuario Arqueológico de Andalucía, Vol. II: 370-376. Sevilla.

NOCETE, F., ORIHUELA, A. , PEÑA, M. y PERAMO, A. (1993): «Odiel.Un año después (1991-1992). 3000-1000 a.n.e. Formaciones Sociales en Transición: Un modelo de análisis histórico para la contrastación del proceso de jerarquización social». Investigaciones Arqueológicas en Andalucía 1985-1992. Proyectos : 383-400. Huelva.

RAMOS, J. (1990): «Informe de la excavación de urgencia realizada en el asentamiento prehistórico de «El Estanquillo» (San Fernando, Cádiz)». Anuario Arqueológico de Andalucía. Sevilla.

RAMOS, J. (1990-1991): «Ensayo de clasificación analítica de los elementos de hoz», Anales de la Universidad de Cádiz VII-VIII:557-572. Cádiz.

RAMOS, J. (1991): «El Estanquillo, análisis microespacial de un asentamiento de la Edad del Bronce», Revista de Arqueologia 122: 14-23. Madrid,

RAMOS, J. (1993): El hábitat prehistórico de «El Estanquillo» (San Fernando, Cádiz). Fundación Municipal de Cultura. Ayuntamiento de San Fernando.

RAMOS, J. (En prensa): «Talleres líticos de la Prehistoria Reciente en Cádiz», $I^{\circ}$ Congreso Internacional «El Estrecho de Gibraltar»: 203-227. Ceuta. 1991. U.N.E.D. Madrid.

RAMOS, J. , BORJA, F. , SÁEZ, A. , CASTAÑEDA, V. , CEPILLO, J. y PÉREZ, M. (1993): «La ocupación prehistórica de la campiña litoral y banda atlántica de Cádiz. Informe de la campaña de prospecciones arqueológicas de 1992 en San Fernando», Investigaciones Arqueologicas en Andalucía: 353-366. Huelva.

RAMOS, J. , GILES, F. , GUTIÉRREZ, J.M. , SANTIAGO, A. , BLANES, C. , MATA, E. , MOLINA, M.I. y VALVERDE, M. (1991): «Aproximación tecnológica a la transición Neolítico-Calcolítico. El 
taller de Cantarranas (El Puerto de Santa María)». Revista de Historia de El Puerto 8:11-33. El Puerto de Santa María.

RAMOS, J. , GILES, F. , SANTIAGO, A., GUTIÉRREZ, J.M. , VALVERDE, M. y MATA, E. (1992): «Explotación de los recursos líticos en la Prehistoria Reciente de Cádiz», Revista de Arqueología 136: 6-17. Madrid.

SCHUBART, H. (1975): Die kultur der Bronzezeit im Südwesten der Iberischen Halbinsel. Madrider Forschungen 9. Berlin.

SERNA, M.R. , ESCACENA, J.L. y AUBET, M.E. (1984): «Nuevos datos para una definición del Bronce Antiguo y Pleno en el Bajo Guadalquivir». Early Settlement in the Western Mediterranean Islands and the Peripheral Areas. B.A.R. International Series, 229: 1051-1073. Oxford.

VALVERDE, M. (1991): «Aproximación a la industria lítica postpaleolítica del taller de Cantarranas (El Puerto de Santa María, Cádiz)». Revista de Historia de El Puerto 7: 11-26. El Puerto de Santa María.

VALVERDE, M. (1993): El taller de Cantarranas (El Puerto de Santa María, Cádiz). Un ejemplo para la transición Neolítico-Calcolítico. Servicio de Publicaciones. Universidad de Cádiz.

ZAZO, C. (1989): «Los depósitos marinos cuaternarios en el golfo de Cádiz», El Cuaternario en Andalucía Occidental. A.E.Q.U.A. Monografías 1: 113-122. Sevilla.

ZAZO , C. , GOY, J.L. , GARCÍA DE DOMINGO, A. , GONZÁLEZ , J. y HERNAIZ, P.P. (1987): San Fernando. Mapa Geológico de España. I.G.M.E. Hoja 11-46. E. 1: 50.000 . Madrid. 